\title{
Typing of Pneumocystis carinii f. sp. hominis by PCR-SSCP to indicate a high frequency of co- infections
}

\author{
AIMABLE NAHIMANA, DOMINIQUE S. BLANC, PATRICK FRANCIOLI, JACQUES BILLE and \\ PHILIPPE M. HAUSER
}

Centre Hospitalier Universitaire Vaudois, 1011 Lausanne, Switzerland

\begin{abstract}
Broncho-alveolar lavage specimens from patients with Pneumocystis carinii pneumonia were investigated by PCR-single strand conformation polymorphism (SSCP) analysis of four genomic regions for $P$. carinii $\mathbf{f}$. sp. hominis. In all, $32 \%$ of specimens produced two bands (one allele) for each of the four genomic regions, suggesting an infection with a single P.c. hominis type. The other specimens displayed more than two bands for at least one of the four genomic regions, suggesting several theoretical possibilities: co-infections, heterozygosity of diploid or polyploid organisms, or multicopy genes. Quantification of the different alleles and analysis of mixtures of specimens showed that different alleles of a genomic region were most often present in different proportions in a given specimen. In contrast, experiments with plasmid insertion of two alleles resulted in the detection of identical proportions of the two alleles. This suggests that neither heterozygosity of diploid organisms nor multicopy genes are responsible for the presence of two alleles. Unequal proportions are most likely explained by the occurrence of co-infections. The putative co-infecting types could be identified in the majority of specimens.
\end{abstract}

\section{Introduction}

The epidemiology of Pneumocystis carinii f. sp. hominis is still poorly understood. Recently, P.c. hominis obtained from broncho-alveolar lavage (BAL) specimens was used to develop a typing method consisting of the amplification of four variable regions of the genome, followed by the detection of the polymorphisms by the single-strand conformation polymorphism technique (SSCP) $[1,2]$. A variable region amplified from a BAL specimen generated either two bands (simple SSCP pattern) or more than two bands (complex pattern). A simple pattern corresponds to a single allele of the genomic region (each band corresponds to one of the two single strands of the PCR product). The presence of more than two bands (complex pattern) indicates the presence of several alleles for a given region [1]. This could be due to (i) co-infection with several P.c. hominis types, (ii) heterozygosity of polyploid organisms, (iii) heterozygosity of diploid organisms, or (iv) multicopy genes with

Received 18 Nov. 1999; revised version received 10 Jan. 2000; accepted 7 Feb. 2000.

Corresponding author: Dr P. M. Hauser (e-mail: Philippe. Hauser@chuv.hospvd.ch). variation between copies, or a combination of these factors [3].

The present study investigated the frequency of the complex SSCP patterns as well as their cause by analysis of the proportions of the alleles within them.

\section{Materials and methods}

Typing of four variable genomic regions by PCRSSCP

Specimens from patients with $P$. carinii pneumonia (PCP) were collected from 1989 to 1998. The four variable genomic regions analysed by PCR-SSCP for typing were: the internal transcribed spacer no. 1 of the nuclear rRNA genes operon (ITS1), the intron of the nuclear 26S rRNA gene (26S), the variable region of the mitochondrial 26S rRNA gene $(\mathrm{mt} 26 \mathrm{~S})$ and the region surrounding the intron no. 6 of the $\beta$-tubulin gene ( $\beta$-tub). Procedures for typing by PCR-SSCP were performed as described previously [1], except that the QIAamp Blood Kit (Qiagen GmbH, Hilden, Germany) was used for extraction of DNA from BAL specimens. 


\section{Quantification of the proportions of different alleles within complex SSCP patterns}

Silver-stained SSCP gels were scanned and the density of each SSCP band of the complex patterns was measured with the NIH Image Analysis Software version 1.61 (W. Rasband, National Institutes of Health, USA).

\section{PCR-SSCP analysis of DNA samples containing an identical proportion of two alleles of a genomic region}

Two alleles of a given genomic region were introduced into the polylinker of a single plasmid by successive clonings. The PCR product from a given BAL specimen was first cloned into the $\operatorname{SrfI}$ site of plasmid pCRScript $^{\mathrm{TM}}$ and introduced in Escherichia coli XL1-blue $\mathrm{MRF}^{\prime}$ with the Amp SK(+) Cloning Kit (Stratagene, La Jolla, USA). The plasmid was extracted according to Del Sal et al. [4] and cut at its EcoRV site where the second allele was inserted. The plasmid carrying the two alleles was cut at the same time with EcoRI (or Bam HI for mt26S), SalI and SacI to separate the two alleles on different DNA fragments. About $1 \mathrm{ng}$ of digested plasmid was used for each PCR-SSCP analysis as described above.

\section{Results}

\section{Analysis of BAL specimens from PCP patients}

A total of 350 BAL specimens collected from 1989 to 1998 was analysed by the multi-target PCR-SSCP typing method $[1,2]$. Two or three simple patterns made of two bands (one allele) were identified for each genomic region (A, B, C; Fig. 1). Complex SSCP patterns corresponding to the superimposition of two, rarely three, of these simple patterns were also observed (for example A,B; $\underline{A}, C$; Fig. 1). The SSCP results of the 350 specimens can be assigned to six different categories, according to the number and proportions of the alleles (detailed results not shown, but available on request). Examples of these six categories chosen randomly are shown in Table 1. A number of specimens produced a simple pattern for each of the four genomic regions, which suggested an infection with a single P.c. hominis type (category I, e.g., specimens 1-3). The rest of the specimens produced a complex pattern for at least one of the four genomic regions (categories II-VI, e.g., specimens 4-22). The present study investigated the significance of the complex SSCP patterns, i.e., the presence of several alleles of one or more genomic regions in a single BAL specimen.

\section{Proportions of the different alleles within the complex SSCP patterns}

About $65 \%$ of the complex SSCP patterns showed a

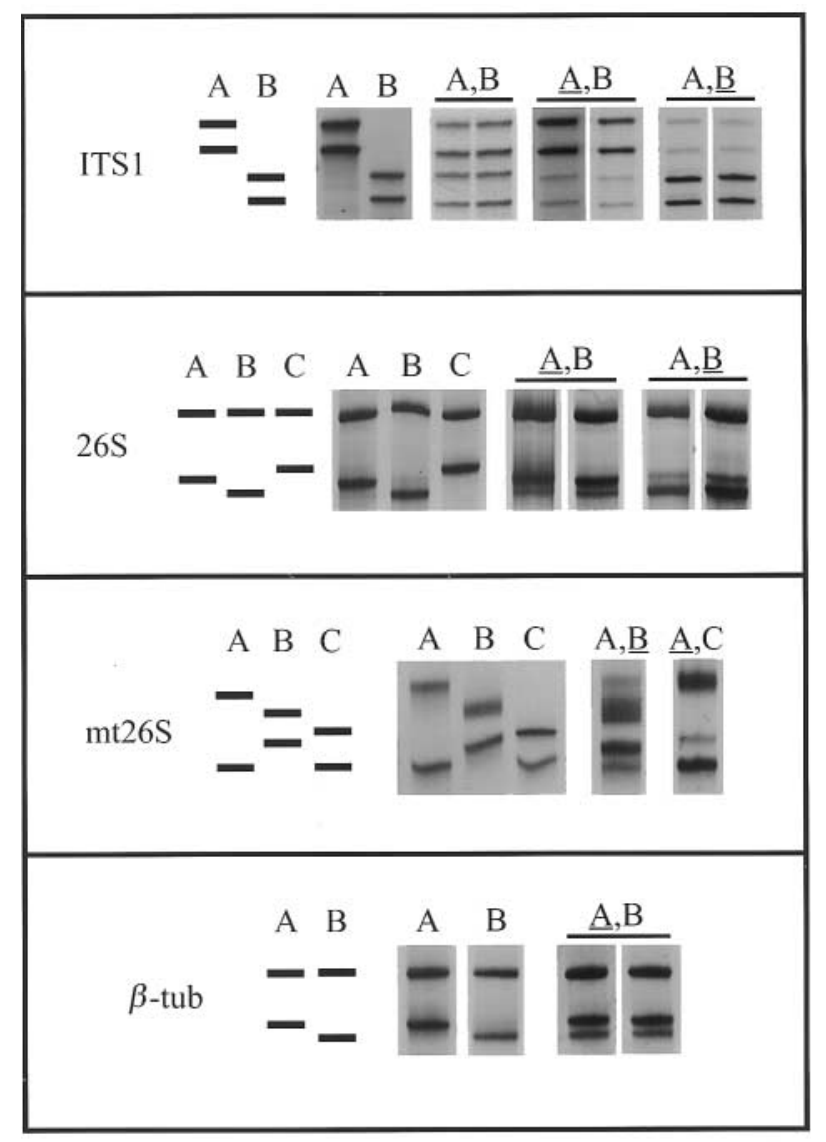

Fig. 1. SSCP patterns produced by the four variable genomic regions amplified to type P.c. hominis. See Materials and methods for abbreviations. Schemes and pictures of the two or three simple SSCP patterns with two bands identified for each region are shown (A, B, C). Examples of complex patterns are also shown (A,B; $\mathrm{A}, \mathrm{C})$. For ITS1, 26S and $\beta$-tub, duplicates of the same specimen are shown (bar above each pair of duplicates). Underlined letters signify the most abundant of the simple patterns within the complex one.

difference in the relative intensity of the silver staining between the two simple patterns (alleles) which was highly reproducible in independent PCR-SSCP analyses of the same specimen (see examples of duplicates in Fig. 1). The difference in the relative staining intensity was not due to any difference in the efficiency of silver staining for a given simple pattern, because the simple pattern which was the most stained in certain specimens was stained less in other specimens (Fig. 1, ITS1 and 26S). This suggested that the alleles were most often present in different proportions within the complex patterns.

\section{PCR-SSCP analysis of mixtures of specimens}

The study investigated whether the proportions of the alleles within the complex patterns reflected those present in the BAL specimen. The DNA preparations of specimens 3 and 5 (Table 1) producing different ITS1 and $\beta$-tub simple SSCP patterns were mixed in different proportions, and the PCR-SSCP procedure was applied to the mixtures. As shown in Fig. 2, the 
Table 1. Examples of the six categories observed among 350 BAL specimens analysed by PCR-SSCP of four variable regions of P.c. hominis genome

\begin{tabular}{|c|c|c|c|c|c|c|c|}
\hline \multirow{2}{*}{$\begin{array}{l}\text { Specimen } \\
\text { no.* }\end{array}$} & \multirow[b]{2}{*}{ Category } & \multirow{2}{*}{$\begin{array}{l}\text { Percent of } \\
\text { specimens }\end{array}$} & \multicolumn{4}{|c|}{ SSCP pattern ${ }^{\dagger}$} & \multirow{2}{*}{$\begin{array}{l}\text { Combination of } \\
\text { simple patterns } \\
\text { (P.c. hominis type })\end{array}$} \\
\hline & & & ITS1 & $26 \mathrm{~S}$ & $\mathrm{mt} 26 \mathrm{~S}$ & $\beta$-tub & \\
\hline 1 & I & \multirow{3}{*}{32} & A & $\mathrm{C}$ & A & A & ACAA \\
\hline 2 & I & & B & B & A & B & $\mathrm{BBAB}$ \\
\hline 3 & I & & A & A & $\mathrm{C}$ & B & $\mathrm{AACB}$ \\
\hline 4 & II & \multirow{3}{*}{21} & $\mathrm{~A}, \mathrm{~B}$ & A & A & A & AAAA, BAAA \\
\hline 5 & II & & $\mathrm{B}$ & B & $\mathrm{A}, \mathrm{B}$ & A & $\overline{\mathrm{BBAA}}, \mathrm{BBBA}$ \\
\hline 6 & II & & A & $\underline{\mathrm{A}}, \mathrm{C}$ & A & A & $\underline{\mathrm{AAAA}}$, ACAA \\
\hline 7 & III & \multirow{9}{*}{24} & $\mathrm{~A}, \underline{\mathrm{B}}$ & $\mathrm{A}, \underline{\mathrm{B}}$ & $\mathrm{A}, \underline{\mathrm{B}}$ & A & AAAA, BBBA \\
\hline 8 & III & & $\underline{\mathrm{A}}, \overline{\mathrm{B}}$ & $\mathrm{A}, \overline{\mathrm{B}}$ & A, $\underline{\bar{C}}$ & $\mathrm{~B}, \underline{\mathrm{C}}$ & $\mathrm{AACC}, \overline{\mathrm{BBAB}}$ \\
\hline 9 & III & & $\overrightarrow{\mathrm{A}}, \underline{\mathrm{B}}$ & $\mathrm{B}$ & $\mathrm{A}, \underline{\mathrm{B}}$ & $\underline{\mathrm{B}}, \overline{\mathrm{C}}$ & $\overline{\mathrm{ABAC}}, \underline{\mathrm{BBBB}}$ \\
\hline 10 & III & & $\underline{\mathrm{A}}, \overline{\mathrm{B}}$ & $\underline{\mathrm{A}}, \mathrm{B}$ & $\underline{\mathrm{A}}, \overline{\mathrm{C}}$ & A & AAAA,$\overline{\mathrm{BBCA}}$ \\
\hline 11 & III & & $\mathrm{A}$ & $\mathrm{B}$ & $\underline{\overrightarrow{\mathrm{A}}}, \mathrm{C}$ & $\mathrm{A}, \underline{\mathrm{B}}$ & $\overline{\overline{\mathrm{ABAB}}}, \mathrm{ABCA}$ \\
\hline 12 & III & & A & $\mathrm{A}, \underline{\mathrm{B}}$ & $\overrightarrow{\mathrm{A}}, \mathrm{C}$ & $\mathrm{A}, \overline{\mathrm{B}}$ & $\overline{\mathrm{AACB}}, \mathrm{ABAA}$ \\
\hline 13 & III & & A & $\mathrm{A}^{-}$ & $\overrightarrow{\mathrm{A}}, \underline{\mathrm{B}}$ & $\overline{\mathrm{A}}, \underline{\mathrm{B}}$ & AAAA, $\overline{\overline{\mathrm{AABB}}}$ \\
\hline 14 & III & & A & $\underline{\mathrm{A}}, \mathrm{B}$ & $\underline{\mathrm{A}}, \overline{\mathrm{C}}$ & $\underline{\mathrm{A}}, \overline{\mathrm{B}}$ & $\underline{\mathrm{AAAA}}, \overline{\mathrm{ABCB}}$ \\
\hline 15 & III & & $\underline{\mathrm{A}}, \mathrm{B}$ & $\underline{\mathrm{A}}, \mathrm{B}$ & $\mathrm{A}, \underline{\mathrm{B}}$ & $\mathrm{B}$ & $\underline{\mathrm{AABB}}, \mathrm{BBAB}$ \\
\hline 16 & IV & \multirow{3}{*}{2} & $\mathrm{~A}, \mathrm{~B}$ & $\mathrm{~A}, \mathrm{~B}$ & A & $\mathrm{A}, \mathrm{B}$ & \multirow{3}{*}{$?$} \\
\hline 17 & IV & & A,B & A,B & $\mathrm{B}, \mathrm{C}$ & $\mathrm{A}, \mathrm{B}$ & \\
\hline 18 & IV & & A & $\mathrm{C}$ & $\mathrm{A}, \mathrm{B}$ & $\mathrm{A}, \mathrm{B}$ & \\
\hline 19 & $\mathrm{~V}$ & \multirow{3}{*}{11} & $\underline{\mathrm{A}}, \mathrm{B}$ & $\mathrm{B}$ & $\underline{\mathrm{A}}, \mathrm{B}$ & $\mathrm{A}, \mathrm{B}$ & \multirow{3}{*}{$?$} \\
\hline 20 & $\mathrm{~V}$ & & $\overrightarrow{\mathrm{A}}, \mathrm{B}$ & $\mathrm{A}, \mathrm{B}$ & $\mathrm{A}, \underline{\mathrm{C}}$ & A,B & \\
\hline 21 & $\mathrm{~V}$ & & A & $\mathrm{A}, \underline{\mathrm{B}}$ & $\underline{\mathrm{A}}, \overline{\mathrm{C}}$ & $\mathrm{A}, \mathrm{B}$ & \\
\hline 20 & VI & \multirow{3}{*}{10} & $\mathrm{~A}, \underline{\mathrm{B}}$ & $\underline{\mathrm{A}}, \mathrm{B}, \mathrm{C}$ & $\underline{\mathrm{A}}, \mathrm{C}$ & A & \multirow{3}{*}{$?$} \\
\hline 21 & VI & & $\mathrm{A}, \overline{\mathrm{B}}$ & $\overline{\mathrm{A}}, \underline{\mathrm{B}}, \mathrm{C}$ & $\overline{\mathrm{A}, \mathrm{B}, \underline{\mathrm{C}}}$ & $\underline{\mathrm{A}}, \mathrm{B}$ & \\
\hline 22 & VI & & $\underline{\mathrm{A}}, \overline{\mathrm{B}}$ & $\mathrm{A}, \underline{\mathrm{B}}$ & $\mathrm{A}^{-}$ & $\overrightarrow{\mathrm{A}, \mathrm{B}, \mathrm{C}}$ & \\
\hline
\end{tabular}

* Specimens were from 18 HIV-positive and 4 HIV-negative patients (nos. 7, 11, 14, 17).

${ }^{\dagger}$ According to Fig. 1. Underlined letters signify the most abundant of the simple patterns within the complex one, as revealed by silver staining.

WUnderlined combinations signify the most abundant putative co-infecting type according to the proportions of the alleles within the complex SSCP patterns (see text).

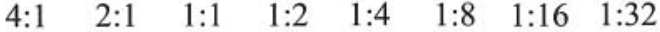

ITS1

$\beta$-tub

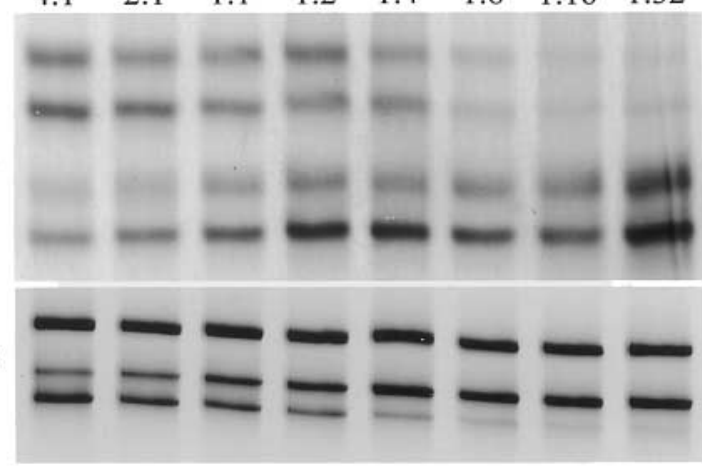

Fig. 2. PCR-SSCP analysis of mixtures of specimens 3 and 5 (Table 1) producing different ITS1 and $\beta$-tub simple patterns. Specimens were mixed in the proportions indicated above the lanes, and the ITS1 and $\beta$-tub region were analysed. Specimen 3 produced ITS simple pattern $\mathrm{A}$ and $\beta$-tub pattern $\mathrm{B}$ (more abundant in mixture 4:1), whereas specimen 5 produced patterns $B$ and $A$ respectively (more abundant in mixture 1:32).

PCR-SSCP method detected the variation of the proportions of the two alleles in the mixture, and the proportion of each DNA added in the mixture was reflected in the two complex patterns produced. Thus, the results show that the PCR-SSCP technique with silver staining of the gel allows a rough quantification of the proportions of the different alleles of a genomic region in a specimen and that these proportions are most often different.

Quantification of the proportions of the different alleles within several complex patterns produced by single specimens

An experiment was performed to test the hypothesis regarding co-infections. The experiment shown in Fig. 2 suggested that, if a specimen corresponds to a coinfection, the less abundant simple patterns within all complex patterns would derive from the less abundant co-infecting type, and the more abundant simple patterns from the co-infecting type present in the majority. Consequently, the proportion of the less abundant simple pattern would be similar for all complex patterns produced by a given specimen. Analysis of nine specimens produced several complex patterns, all made up of different proportions of the two simple patterns (Table 1, specimens 7-15). The PCRSSCP of each genomic region was repeated twice and the relative proportion of the less abundant simple pattern within each complex pattern was measured by quantification of the band intensities. The data obtained are shown in Table 2. The proportions of the less 
Table 2. Relative proportion of the less abundant simple SSCP pattern within the complex patterns produced by single specimens*

\begin{tabular}{lccccc}
\hline & \multicolumn{4}{c}{ Proportion in genomic region } & \\
\cline { 2 - 5 } Specimen no. & ITS1 & $26 \mathrm{~S}$ & $\mathrm{mt26S}$ & $\beta$-tub & \multirow{2}{*}{ Mean (SD) } \\
\hline 7 & 0.34 & 0.30 & 0.29 & $\mathrm{SP}^{\dagger}$ & $0.33(0.05)$ \\
& 0.28 & 0.35 & 0.40 & & \\
8 & 0.10 & 0.14 & 0.11 & 0.17 & $0.15(0.05)$ \\
& 0.14 & 0.24 & 0.09 & 0.19 & \\
9 & 0.43 & $\mathrm{SP}$ & 0.43 & 0.34 & $0.41(0.05)$ \\
& 0.48 & & 0.38 & 0.38 & \\
10 & 0.24 & 0.31 & 0.23 & $\mathrm{SP}$ & $0.26(0.04)$ \\
& 0.24 & 0.32 & 0.22 & & \\
11 & $\mathrm{SP}$ & $\mathrm{SP}$ & 0.21 & 0.25 & $0.24(0.05)$ \\
& & & 0.19 & 0.31 & \\
12 & $\mathrm{SP}$ & 0.39 & 0.45 & 0.32 & $0.40(0.05)$ \\
& & 0.43 & ND & 0.41 & \\
13 & $\mathrm{SP}$ & $\mathrm{SP}$ & 0.28 & 0.38 & $0.35(0.06)$ \\
& & & ND & 0.38 & \\
14 & $\mathrm{SP}$ & 0.19 & 0.20 & 0.16 & $0.21(0.05)$ \\
& & 0.21 & 0.30 & 0.21 & \\
15 & 0.28 & 0.32 & ND & SP & $0.27(0.06)$ \\
& 0.29 & 0.19 & ND & & \\
\hline
\end{tabular}

$\mathrm{ND}$, not determined because of insufficient resolution of the SSCP bands.

* SSCP gels were scanned and each simple SSCP pattern within the complex patterns described in Table 1 was quantified by the NIH Image Analysis Software. The proportion of the less abundant simple pattern relative to the sum of the two simple patterns is given. For each specimen, PCR-SSCP of each genomic region was done in duplicate.

${ }^{\dagger}$ Simple SSCP pattern for the region investigated (see Table 1).

abundant simple pattern of each given specimen were relatively homogeneous, a result compatible with the hypothesis of co-infections.

In natural co-infections, the proportions of the coinfecting types are expected to vary from specimen to specimen. Thus, the mean proportion of the less abundant pattern should vary significantly from specimen to specimen. To test this hypothesis, the sets of data obtained for each specimen (Table 2) were compared to each other. The mean proportions of the less abundant pattern were significantly different in the majority of the pairs (22 of 36) (Mann-Whitney test, $\mathrm{p}=0.0007-0.045)$.

\section{PCR-SSCP analysis of DNAs containing an identical proportion of two alleles of a given genomic region}

The previous results suggested that the different alleles of the genomic regions are amplified with a similar efficiency when co-amplified in a single reaction. This would be in agreement with the fact that they differ only by a few base pairs $(2-4 \%$ divergence at the nucleotide sequence level $[1,5])$. To confirm this explanation, two alleles of a genomic region producing different simple SSCP patterns were cloned into the same plasmid and the PCR-SSCP procedure was applied. Four-to-nine independent clones according to the four different genomic regions were analysed. As expected from the fact that the two alleles were equimolar in the reaction, all clones produced complex patterns showing similar staining intensity for the two simple patterns (examples are shown in Fig. 3).

\section{Sensitivity of the detection of an allele present in low amounts}

The experiment shown in Fig. 2 allows an estimation of the sensitivity of the detection of an allele present in low amounts in a mixture of alleles and, thus, of a potential co-infection. Indeed, the less abundant ITS1 and $\beta$-tub simple patterns in the complex patterns are clearly visible up to the mixture at a ratio of $1: 8$. The latter mixture corresponds to a proportion of the less abundant simple pattern of $11 \%$, suggesting that an allele or a putative co-infecting type must represent at
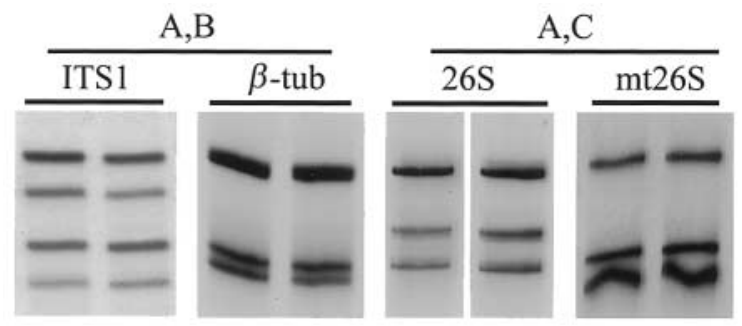

Fig. 3. PCR-SSCP analysis of independent plasmid clones containing two alleles of a genomic region. Each allele produced one of the two simple patterns within each complex pattern A,B or A,C. The ITS1 alleles cloned were those from specimens 3 and 5 (Table 1). All other cloned alleles were described previously [1]. 
least this proportion of the population to be detected by the PCR-SSCP method.

\section{Interpretation of SSCP results and identification of the co-infecting types for typing}

The above results strongly suggest that the presence of several alleles of a genomic region in a specimen is due to a co-infection, raising the issue of the identification of the putative co-infecting types. For typing purposes, six different situations can be distinguished as follows.

(i) Specimens producing four simple SSCP patterns corresponding to the four genomic regions (category I, examples are shown in Table 1). These specimens are presumably infected with a single P.c. hominis type and each combination of four simple patterns defines a type.

(ii) Specimens producing only one complex SSCP pattern made up of two simple patterns (category II). The identification of the putative co-infecting types is easy for these specimens. Indeed, the two types produced the same simple pattern for three genomic regions, but a different one for the last genomic region.

(iii) Specimens producing two-to-four complex SSCP patterns which are all made of two simple patterns present in different proportions (category III). The putative co-infecting types can also be identified for these specimens, the less abundant simple patterns would derive from the less abundant co-infecting type, and the more abundant from the second type (Fig. 2).

(iv) Specimens producing several complex SSCP patterns all with similar proportions of the two simple patterns (category IV). This suggests that the presence of two co-infecting types in similar proportions. However, the types cannot be identified because there are theoretically many different possible combinations of four simple patterns.

(v) Specimens producing two-to-four complex SSCP patterns with proportions of the two simple patterns that were similar for some genomic regions but different for others (category V). The putative coinfecting types cannot be identified. The results of these specimens could be explained by the presence of more than two types.

(vi) Specimens producing at least one complex SSCP pattern made of three simple patterns (category VI). These are presumably infected with at least three types which cannot be identified.

Interestingly, the most prevalent P.c. hominis types identified in the putative co-infections were also the most prevalent in the infections with a single type.

\section{Discussion}

In all, $68 \%$ of the BAL specimens from patients with PCP produced complex SSCP patterns reflecting the presence of two, rarely three, alleles of the genomic region. The proportions of the alleles were most often different as revealed by silver staining. Analysis of mixtures of DNAs containing two alleles of a genomic region in different proportions and plasmid DNAs containing equimolar amounts of two alleles showed that the proportions of the alleles within the complex SSCP patterns reflected those present in the specimen. This finding suggests that the presence of several alleles in a single specimen was not due to heterozygosity of diploid organisms, nor to the presence of multiple copies of the genes, because these hypotheses imply identical proportions of the alleles. The fact that multiple copies of genes are not responsible for the complex patterns is in agreement with the finding of a single copy per genome of the genes used to type P.c. hominis $[6,7]$.

Co-infections with several types or heterozygosity in polyploid organisms are two possibilities that may account for the complex SSCP patterns, because they could both result in different proportions of the alleles in a specimen. Quantification of the SSCP bands showed that when several alleles were present in one specimen they were present in similar proportions for all genomic regions. This is in agreement with the hypothesis of co-infections: the less abundant simple pattern within all the complex patterns would derive from the less abundant co-infecting type, and the more abundant simple pattern from the more abundant coinfecting type. Polyploidy has been described in fungi $[8,9]$, but the results would be more difficult to explain with this hypothesis. The less abundant patterns could derive from one of the chromosome sets of polyploid cells, and the more abundant patterns from the other sets. However, this possibility would require an additional mechanism to account for the fact that the proportion of the alleles of the mitochondrial genomic region (mt26S) is similar to those of the alleles of the nuclear genomic regions (ITS1, 26S and $\beta$-tub), which appears unlikely.

The hypothesis of co-infections is strongly favoured by the following facts. First, the occurrence of coinfections would be reminiscent of the situation observed in $P$. carinii infections of rats $[10,11]$ and ferrets [12]. Second, haploidy of P.c. hominis is suggested by the recent demonstration of the haploidy of the trophozoites and intracystic bodies of $P$. $c$. carinii, the special form infecting rats [13]. Third, as observed in Schizosaccharomyces pombe polyploid strains [14], more than two chromosome sets per cell would probably lead to an increase of the cellular or nuclear size in P. c. hominis, or both, as compared with $P$. c. carinii, and this has not been reported in electron microscopy investigations of the morphology of $P$. $c$. 
hominis cells $[15,16]$. Taken together, all these observations strongly favour the hypothesis of coinfections. The occurrence of co-infections in human patients has already been suggested in several studies $[12,17-21]$, although at a frequency of only $10-35 \%$. As discussed previously [2], there are two possibilities, which are not mutually exclusive, to explain this difference. First, the sensitivity of silver staining used in SSCP allows the detection of a co-infecting type if it represents at least $11 \%$ of the micro-organism population, whereas this proportion rises to at least $20 \%$ for the other methods used to detect co-infections. Second, the difference might reflect true differences in the proportion of co-infection in the population analysed.

In most cases careful analysis of the SSCP results of one specimen allows the identification of each putative co-infecting type as well as the abundance of the types causing that infection. The minimal proportion of the micro-organism population that a putative co-infecting type must represent to be detected was estimated to be $11 \%$. These results should allow more precise epidemiological studies to be performed.

This work was supported by grant nos. 32-53994.98 from the Swiss National Fund, and 94-7213 and 97-7299 from the Swiss National Program on AIDS Research. We thank Arlette Cruchon for excellent technical assistance and Paul Majcherczyck for critical reading of the manuscript.

\section{References}

1. Hauser PM, Francioli P, Bille J, Telenti A, Blanc DS. Typing of Pneumocystis carinii f. sp. hominis by single-strand conformation polymorphism of four genomic regions. J Clin Microbiol 1997; 35: 3086-3091.

2. Hauser PM, Blanc DS, Bille J, Francioli P. Typing methods to approach Pneumocystis carinii genetic heterogeneity. FEMS Immunol Med Microbiol 1998; 22: 27-35.

3. Hauser PM, Blanc DS, Telenti A, Nahimana A, Bille J, Francioli P. Potential coinfections complicate typing of Pneumocystis carinii f. sp. hominis. J Clin Microbiol 1998; 36: 3111 .

4. Del Sal G, Manfioletti G, Schneider C. A one-tube plasmid DNA mini-preparation suitable for sequencing. Nucleic Acids Res 1988; 16: 9878 .

5. Stringer JR. Pneumocystis carinii - what is it, exactly? Clin Microbiol Rev 1996; 9: 489-498.

6. Nahimana A. Determination of the copy number per genome of the genes used to type Pneumocystis carinii sp. f. hominis. Master thesis, University of Lausanne, Switzerland. 1998.

7. Tang X, Bartlett MS, Smith JW, Lu J-J, Lee C-H. Determination of copy number of rRNA genes in Pneumocystis carinii $\mathrm{f}$. sp. hominis. J Clin Microbiol 1998; 36: 2491-2494.

8. Buttner P, Koch F, Voigt $\mathrm{K}$ et al. Variations in ploidy among isolates of Botrytis cinerea: implications for genetic and molecular analyses. Curr Genet 1994; 25: 445-450.

9. Guijo S, Mauricio JC, Salmon JM, Ortega JM. Determination of the relative ploidy in different Saccharomyces cerevisiae strains used for fermentation and 'flor' film ageing of dry sherry-type wines. Yeast 1997; 13: 101-117.

10. Cushion MT, Zhang J, Kaselis M, Giuntoli D, Stringer SL, Stringer JR. Evidence for two genetic variants of Pneumocystis carinii coinfecting laboratory rats. J Clin Microbiol 1993; 31: $1217-1223$.

11. Hong S-T, Steele PE, Cushion MT, Walzer PD, Stringer SL, Stringer JR. Pneumocystis carinii karyotypes. J Clin Microbiol 1990; 28: 1785-1795.

12. Banerji S, Lugli EB, Miller RF, Wakefield AE. Analysis of genetic diversity at the arom locus in isolates of Pneumocystis carinii. J Eukaryot Microbiol 1995; 42: 675-679.

13. Wyder MA, Rasch EM, Kaneshiro ES. Quantitation of absolute Pneumocystis carinii nuclear DNA content. Trophic and cystic forms isolated from infected rat lungs are haploid organisms. J Eukaryot Microbiol 1998; 45: 233-239.

14. Molnar M, Sipiczki M. Polyploidy in the haplontic yeast Schizosaccharomyces pombe: construction and analysis of strains. Curr Genet 1993; 24: 45-52.

15. Ishida T, Matsui Y, Matsumura Y, Furutani M. Ultrastructural observation of Pneumocystis carinii in bronchoalveolar lavage fluid from non-AIDS patients with $P$. carinii pneumonia. Chest 1994; 105: 1342-1346.

16. Campbell WG. Ultrastructure of Pneumocystis in human lung. Life cycle in human pneumocystosis. Arch Pathol 1972; 93: 312-324.

17. Lee C-H, Helweg-Larsen J, Tang $\mathrm{X}$ et al. Update on Pneumocystis carinii f. sp. hominis typing based on nucleotide sequence variations in internal transcribed spacer regions of rRNA genes. J Clin Microbiol 1998; 36: 734-741.

18. Lu JJ, Bartlett MS, Shaw MM et al. Typing of Pneumocystis carinii strains that infect humans based on nucleotide sequence variations of internal transcribed spacers of rRNA genes. J Clin Microbiol 1994; 32: 2904-2912.

19. Tsolaki AG, Miller RF, Underwood AP, Banerji S, Wakefield AE. Genetic diversity at the internal transcribed spacer regions of the rRNA operon among isolates of Pneumocystis carinii from AIDS patients with recurrent pneumonia. J Infect Dis 1996; 174: 141-156.

20. Latouche S, Poirot J-L, Bernard C, Roux P. Study of internal transcribed spacer and mitochondrial large-subunit genes of Pneumocystis carinii hominis isolated by repeated bronchoalveolar lavage from human immunodeficiency virus-infected patients during one or several episodes of pneumonia. J Clin Microbiol 1997; 35: 1687-1690.

21. Keely SP, Stringer JR, Baughman RP, Linke MJ, Walzer PD, Smulian AG. Genetic variation among Pneumocystis carinii hominis isolates in recurrent pneumocystosis. J Infect Dis 1995; 172: 595-598. 